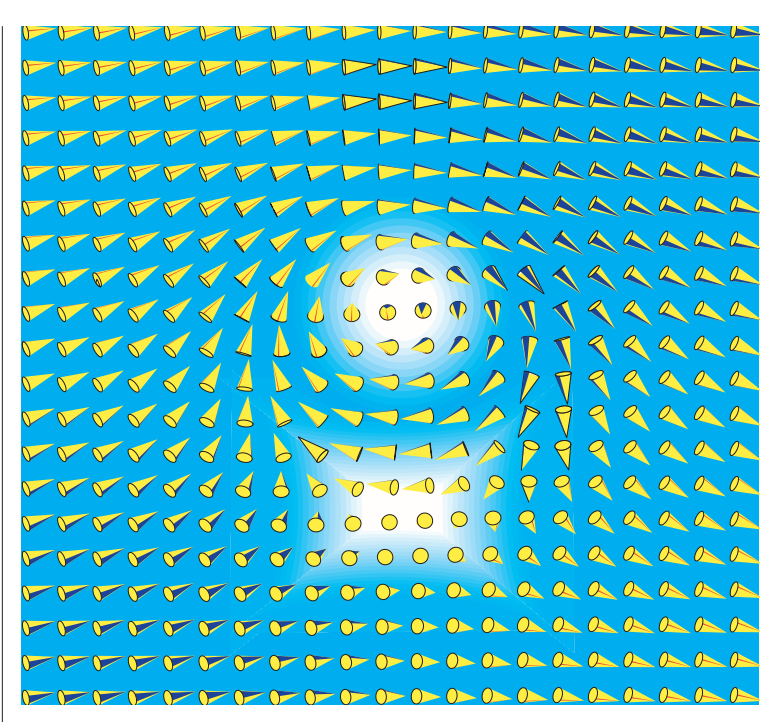

Figure 1 The doubly quantized vortex observed by Blaauwgeers and colleagues. The cones indicate the direction of the Cooper-pair angular momentum. The angular momentum makes a full turn in direction on going round the circular and hyperbolic cores. The circulation is given by the rotation of the cones about their axes. This makes a full $4 \pi$, double turn, following round the perimeter. because the direction of both the nuclear and the orbital spins, as well as the phase of the wavefunction, can make a $2 n \pi$ change around the axis, then many different vortex structures are possible and the energy restriction on all but singly quantized vortices is lifted.

In their experiment, Blaauwgeers and colleagues identify a doubly quantized vortex (that is, $n=2$ ) in superfluid ${ }^{3} \mathrm{He}$ from its NMR signature. This vortex is shown in Fig. 1. The cones point in the direction of the orbital angular momentum of the Cooper pairs. The circulation depends on the angle of rotation of the coloured bands around the cone axis. Following around the perimeter of Fig. 1, the cones make two full turns, corresponding to a $4 \pi$ rotation.

In ${ }^{3} \mathrm{He}$, several quantities are changing simultaneously, so there is no singularity along the axis (the air-filled core in the plughole version) and all quantities vary smoothly across the axis. The vortex is also rather large, of order 10 micrometres in diameter, or not far off the size of a small snowflake. Indeed, this is a new snowflake, a natural structure having its own beautiful symmetry and the added bonus of stable symmetrical flow. Because superfluid ${ }^{3} \mathrm{He}$ can only exist below $2.6 \mathrm{mK}$ above absolute zero, these new 'snowflakes' are even more fugitive than those of ice.

Vortices in this superfluid are not simply aesthetic structures in an arcane fluid. The symmetry of the wavefunction of superfluid ${ }^{3} \mathrm{He}$ mimics surprisingly closely the metric of the Universe. Vortices are the superfluid analogue of cosmic strings. In the Universe, cosmic strings, if they indeed exist, are the relics of cosmic turbulence frozen into space-time by rapid phase transitions in the Universe shortly after the big bang. In the experiment of Blaauwgeers et al. the vortices are under strict control and can be injected one by one into the liquid as the rotation rate is increased. Indeed, this is how the experiment is done. Until we find real cosmic strings to study, vortices in superfluid ${ }^{3} \mathrm{He}$ are the best substitute.

George Pickett is in the Department of Physics,

University of Lancaster, Lancaster LA1 4YB, UK.

e-mail: g.pickett@lancaster.ac.uk

\title{
Bacterial genomics
}

\section{$A B C$ of meningococcal diversity}

\author{
Ian M. Feavers
}

$\mathrm{M}$ eningococcal disease is dreaded by parents and medical practitioners alike for its rapid onset and the difficulty of obtaining a timely and accurate diagnosis. Although the causative bacterium, Neisseria meningitidis (Fig. 1, overleaf), is responsive to antibiotic therapy, these features of the disease pose a particular problem for treatment. Prevention of the disease through effective immunization is thus a more attractive alternative than therapy. For example, during the past five months new vaccines have been introduced into the UK vaccination programme that, for the first time, offer protection to young infants against disease caused by $N$. meningitidis serogroup C. The success of this programme emphasizes the need for the development of vaccines against all pathogenic strains of $N$. meningitidis.

The complete genome sequences of the serogroup A and B organisms are now reported by, respectively, Parkhill et al. on page 502 of this issue ${ }^{1}$ and Tettelin et al. in
Science $^{2}$. These papers represent a milestone in meningococcal research. As well as providing the basis for future studies of the biology and the disease-causing mechanisms of this important pathogen, they highlight the potential of bacterial genomics to provide mankind with new solutions to intractable infectious diseases. However, the data also show us that a headlong rush to evaluate new vaccine candidates should be tempered with caution. Both genomes are replete with an unprecedented number of genetic elements that contribute to both genome fluidity and physical variability, attesting to the adaptability of $N$. meningitidis and its potential to evade the human immune response.

Neisseria meningitidis is a major cause of bacterial meningitis and septicaemia, diseases that all too frequently result in death or disability. Serogroup A organisms are responsible for large epidemics in sub-Saharan Africa, whereas serogroup B and C organisms account for sporadic cases and numerous localized outbreaks worldwide ${ }^{3}$.

Despite the reputation it has earned as an aggressive pathogen, $N$. meningitidis is more frequently found as a harmless commensal - an organism that lives with, but does not harm, the host - in the human nose and pharynx. It has been estimated that, at any given time, between $5 \%$ and $10 \%$ of people carry the organism. Sporadic cases of disease occur at the much lower annual rates of 1 to 5 per 100,000. The bacterium lives only in humans, passing from one person to another in aerosols; there are no other animal or environmental niches that it can colonize. It is therefore highly adapted to living in humans and has evolved a variety of mechanisms to evade our defences, including the ability to produce polysaccharide capsules that provoke a poor immune response, and very variable cell-surface proteins that represent an ever-changing target for the immune system.

The genetic basis for the adaptability of $N$. meningitidis is evident from the analyses of the genome sequence data by Parkhill et $a .^{1}$ and Tettelin et al. ${ }^{2}$. These data reveal an abundance of diverse repeat sequences in each meningococcal strain. These sequences are likely to have a key role in mediating variation within each genome and hence in promoting the adaptability of the organism. A large proportion of repeat sequences is associated with genes encoding cell-surface structures that are involved in the interaction of the organism with the host and thus represent possible targets for vaccine development. Tettelin et al. ${ }^{2}$ estimate that the expression of as many as 65 genes in the serogroup B strain MC58 might be altered by the gain or loss of nucleotides within iterative sequence motifs as a result of inaccurate DNA replication. The effect of such sequence changes on the expression of these genes and, ultimately, on the synthesis of the proteins they encode, 
provides the bacterium with the ability to switch rapidly and reversibly between a vast number of possible combinations of physical attributes.

Other types of repetitive DNA allow further diversity, mediating genomic recombination both within and between different meningococcal strains. Recombination between strains makes use of the natural ability of the meningococcus to acquire DNA from its environment and is undoubtedly helped by the prevalence of DNA-uptake sequences - specific nucleotide sequences that enable the acquisition of DNA by other meningococci-in the genomes. All of these mechanisms provide the bacterium with the means to overcome the host's immune response to both infection and vaccination. Such concerns have been raised recently with regard particularly to the ability of meningococcal strains to exchange the genes responsible for biosynthesis of their capsules ${ }^{4}$.

Fortunately, as well as highlighting the extent of the problem, meningococcal genomics also provides possible solutions. Pizza et $a .^{5}$ have screened the serotype B genome for genes encoding cell-surface antigens that are highly conserved in all meningococcal strains. Their aim is to make use of these antigens as vaccine components. This approach, although giving encouraging results in an animal model $l^{5}$, must now be proved in clinical studies. The comparison of different meningococcal genomes might also reveal genes that distinguish the relatively small number of hypervirulent strains from all other meningococci, and provide the basis for a strategy that specifically targets the properties encoded by such genes.

We do not yet know which strategy, or combination of strategies, will provide an

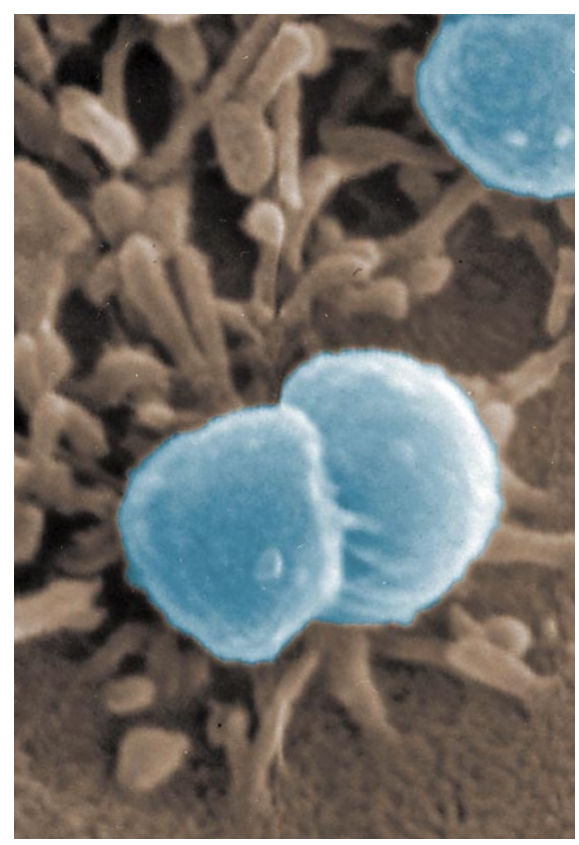

Figure 1 The meningococcus - a bacterium with unprecedented potential for diversity ${ }^{1,2}$. effective solution to the problem of meningococcal disease. But the public availability of the two genome sequences is bound to hasten the development of comprehensive vaccines that offer protection against all virulent meningococci.

Ian M. Feavers is in the Division of Bacteriology, National Institute for Biological Standards and
Control, Blanche Lane, South Mimms, Potters Bar EN6 3QG, UK

\section{e-mail:ifeavers@nibsc.ac.uk}

1. Parkhill, J. et al. Nature 404, 502-506 (2000).

2. Tettelin, H. et al. Science 287, 1809-1815 (2000).

3. Schwartz, B., Moore, P. S. \& Broome, C. V. Clin. Microbiol. Rev 2 (Suppl.), 118-124 (1989).

4. Maiden, M. C. \& Spratt, B. G. Lancet 354, 615-616 (1999).

5. Pizza, M. et al. Science 287, 1816-1820 (2000).

Particle physics

\section{Backyard exotica}

Frank Wilczek

A 11 atoms follow the same body plan. They consist of a small, massive nucleus of positive charge surrounded by a cloud of negative electrons, bound together by the electromagnetic force. One might say that all atoms belong to a single phylum. Hadrons, elementary particles that are governed by the strong nuclear force, are known to populate three extremely well documented phyla. Baryons (such as protons and neutrons) are based on three quarks having different colour charges, one each of red, white and blue. Antibaryons are similarly constructed from triads of antiquarks, and mesons are based on bound quark-antiquark pairs, carrying equal and opposite colour charges. Each of these hadron phyla supports dozens of species. For many years now, physicists have been on the lookout for exotic new species, requiring more hadron phyla. We might now have the first hint from a theoretical analysis by Alford and Jaffe ${ }^{1}$ of something suitably exotic.

What are these new particles? Among the range of possibilities dreamt up by theorists are various types of glueball, made from either two or three colour gluons (the particles that bind quarks together). Or there are centaurons (also called hybrids) made from a quark, an antiquark and a gluon. Finally, we have a hypothetical phylum that does not yet have an accepted name, based on two quarks and two antiquarks. It seems irresistible to call these quarktets. Alford and Jaffe use ingenious computer experiments to argue that some long familiar, but hitherto enigmatic, particles are best interpreted as quarktets ${ }^{1}$.

Hundreds of different strongly interacting particles have been identified. Most are very short-lived (less than $10^{-20}$ seconds), and are observed only as enhancements in particle production rates, known as resonances. The observed properties of many of these particles match up with the predicted properties of different arrangements of quarks and antiquarks conforming to one of the baryon, antibaryon or meson body plans. Within each plan, one has the freedom to choose different properties for the quarks, which can result in states with different total energies (or masses, according to $E=m c^{2}$ ). By this reasoning, the pattern of resonances can be seen as a 'spectrum', wholly analogous to the pattern of stationary states of an atom (as observed from its spectral lines).

The logical development of this line of thought is the quark model ${ }^{2}$. The quark model was cultivated during the 1960 s and early 1970s, finally reaching its most mature form in the so-called bag model ${ }^{3}$. According to the bag model, hadrons consist of relativistic, nearly free quarks (and/or antiquarks) confined to a finite region of space by a universal, constant vacuum pressure. The quark/bag model accounts, semiquantitatively, for an enormous range of data. But it is not a satisfactory fundamental theory. For example, simply trying to compute what happens when bag-hadrons move results in mathematical ambiguities.

The fundamental theory of the strong force, quantum chromodynamics or QCD, emerged from the study of extremely highenergy collisions. QCD accepts quarks and antiquarks as fundamental ingredients, but also requires colour gluons. Indeed, the exchange of colour gluons is fully responsible for the strong interactions between quarks, much as the exchange of photons is responsible for electromagnetic interactions. Unlike the quark/bag model, QCD is a proper, precisely defined fundamental theory.

If correct, QCD should be able to predict the major properties and strong interactions of hadrons. Unfortunately, however, the equations of QCD are very difficult to solve. Computations that strain at the limits of modern, massively parallel computers succeed in producing, within a few per cent accuracy, the masses of a dozen or so of the lowest states of the hadron spectrum ${ }^{4}$. This is a remarkable achievement and confirms the correctness of a beautiful, parameter-free theory ${ }^{5}$. Yet to understand the rest of the hadron spectrum one will need, for the foreseeable future, to build upon older, but more manageable, concepts and models. Within this humbler approach, QCD does not dic- 